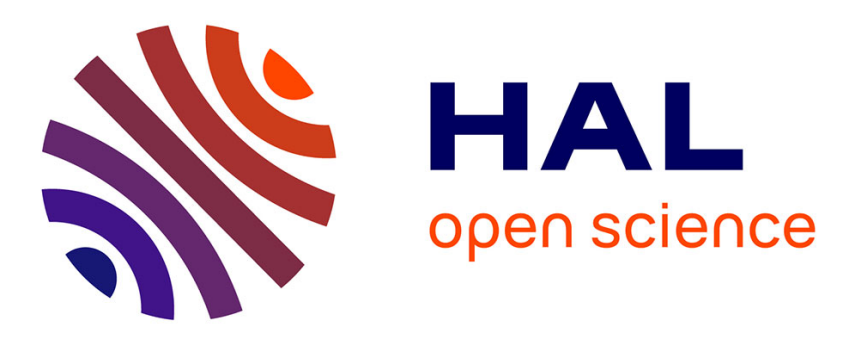

\title{
Passive SHM system for corrosion detection by guided wave tomography
}

Tom Druet, Bastien Chapuis, Manfred Jules, Guillaume Laffont, Emmanuel Moulin

\section{- To cite this version:}

Tom Druet, Bastien Chapuis, Manfred Jules, Guillaume Laffont, Emmanuel Moulin. Passive SHM system for corrosion detection by guided wave tomography. Sensors, Algorithms and Applications for Structural Health Monitoring: IIW Seminar on SHM, Springer, pp.21-29, 2017, IIW Collection book series, 978-3-319-69233-3. 10.1007/978-3-319-69233-3_3 . cea-03285038

\section{HAL Id: cea-03285038 https://hal-cea.archives-ouvertes.fr/cea-03285038}

Submitted on 13 Jul 2021

HAL is a multi-disciplinary open access archive for the deposit and dissemination of scientific research documents, whether they are published or not. The documents may come from teaching and research institutions in France or abroad, or from public or private research centers.
L'archive ouverte pluridisciplinaire HAL, est destinée au dépôt et à la diffusion de documents scientifiques de niveau recherche, publiés ou non, émanant des établissements d'enseignement et de recherche français ou étrangers, des laboratoires publics ou privés. 


\title{
COMMISSION V
}

NDT AND QUALITY ASSURANCE OF WELDED PRODUCTS

V-1670-15

\section{PASSIVE SHM SYSTEM FOR CORROSION DETECTION BY GUIDED WAVE TOMOGRAPHY}

Authors: Tom Druet (a), Bastien Chapuis (a), Manfred Jules (a), Guillaume Laffont (a), and Emmanuel Moulin (b)

\begin{abstract}
:
Guided elastic waves emitted by a sensor and propagating to another one are often used as the physical way of detecting the defect in Structural Health Monitoring (SHM) systems of plate like structures. However, the implementation of SHM systems is restricted in many situations by the necessity to store or to harvest the electric energy necessary to emit the waves. A promising way to overcome this constraint is to use techniques based on the cross-correlations of the ambient noise in place in the structure. The idea is to take advantage of the elastic noise naturally present in the structure (due to engine vibrations or aero-acoustic turbulences on the fuselage of an aircraft for example) in order to avoid the emission of the elastic waves by the SHM system. The complexity of the embedded SHM system is therefore reduced. We present here studies of noise cross-correlation techniques that have been conducted with the aim of doing passive guided wave tomography of extended defects (such as corrosion) using an array of piezoelectric (PZT) transducers. Noise is generated by spraying compressed air on the surface of a thin aluminum plate. Passive measurements are compared to active signals to demonstrate the effectiveness of the cross-correlation technique. Experimental results which come from tomographic time-of-flight imaging algorithms will also be described. Finally, an extension of this technique using Fiber Bragg Gratings (FBG) optic sensors will be presented.
\end{abstract}

IIW Keywords:

NDT, non-destructive testing, structural health monitoring, SHM, tomography 
Passive SHM system for corrosion detection by guided wave tomography

Tom Druet (a), Bastien Chapuis (a), Manfred Jules

(a), Guillaume Laffont (a), and Emmanuel Moulin

(b)

(a) CEA LIST (France)

(b) Université de Valenciennes (France)

\begin{abstract}
Guided elastic waves emitted by a sensor and propagating to another one are often used as the physical way of detecting the defect in Structural Health Monitoring (SHM) systems of plate like structures. However, the implementation of SHM systems is restricted in many situations by the necessity to store or to harvest the electric energy necessary to emit the waves. A promising way to overcome this constraint is to use techniques based on the cross-correlations of the ambient noise in place in the structure. The idea is to take advantage of the elastic noise naturally present in the structure (due to engine vibrations or aero-acoustic turbulences on the fuselage of an aircraft for example) in order to avoid the emission of the elastic waves by the SHM system. The complexity of the embedded SHM system is therefore reduced. We present here studies of noise cross-correlation techniques that have been conducted with the aim of doing passive guided wave tomography of extended defects (such as corrosion) using an array of piezoelectric (PZT) transducers. Noise is generated by spraying compressed air on the surface of a thin aluminum plate. Passive measurements are compared to active signals to demonstrate the effectiveness of the cross-correlation technique. Experimental results which come from tomographic time-of-flight imaging algorithms will also be described. Finally, an extension of this technique using Fiber Bragg Gratings (FBG) optic sensors will be presented.
\end{abstract}

IIW-Thesaurus keywords: NDT, non-destructive testing, structural health monitoring, SHM, tomography

\title{
1 Introduction
}

The Structural Health Monitoring (SHM) consists of embedding sensors in a structure such as an aircraft or a naval ship in order to detect defects (for example cracks or corrosion in metallic materials or delamination in composite materials) before a serious fault occurs in the structure. Guided elastic waves emitted by a sensor and propagating to another one are often used as the physical way to detect defects. In aeronautics, the classical approach generally aims at minimizing the number of sensors to limit the embedded mass as well as the sensors intrusiveness within the structure. Comparisons between current signals and baseline signals are often performed in order to reveal the presence of defects [1]. However, this method may not be robust under certain conditions such as changes in temperature, stress, sensors aging, etc.

A possible strategy to avoid the use of baseline signals can consist in increasing the number of sensors and performing guided wave tomography. Indeed, more relevant physical information is obtained from the structure, making the diagnosis more robust. Moreover tomography algorithms produce images that are much easier to interpret than temporal signals. Implementation of SHM systems with a large number of sensors could be intrusive with the use of piezoelectric transducers. Optical fiber sensors using Fiber Bragg gratings (FBGs) for dynamic strain measurements should allow multiplexing capabilities with low intrusiveness in the structure. However, FBG are generally used only as sensors but not as a source of elastic waves. A promising way to tackle these constraints is to use techniques based on cross-correlation of the ambient acoustic noise present in the structure. It has been shown that, under certain conditions, transient responses between two sensors can be passively estimated from the cross-correlation of ambient noise [2, 3, 4]. The idea is to take advantage of the elastic noise naturally present in the structure (due to engine vibrations or aero-acoustic turbulence on the fuselage of an aircraft for example) in order to avoid the need for emission of the elastic waves by the SHM system.

This paper shows an active tomography image which has been performed with a time-of-flight tomography algorithm using experimental data produced by piezoelectric transducers. Furthermore, a comparison between active signals and passive signals which comes from cross-correlation of ambient 
noise produced by spraying compressed air on an aluminum plate shows that it is possible to detect time-of-flight passively. In Section 2 the tomography algorithm used for this work is presented. Then, in Section 3 the experimental setup used to acquire the data necessary to obtain the tomography images is described. Finally, in Sections 4 and 5 the experiments are presented showing that it is possible to use cross-correlation of ambient noise present in the plate-like structure to get the data required for the tomography algorithm. Several configurations of sensors were studied using piezoelectric transducers, Fiber Bragg Gratings and a combination thereof.

\section{Time-of-Flight Tomography Algorithm}

Images shown in this paper are obtained with a time-of-flight tomography algorithm which uses the Simultaneous Iterative Reconstructive Technique (SIRT) [5]. Straight ray assumption is taken within this framework. Straight ray tomography does not take into account refraction and diffraction. By ignoring diffraction, only defects bigger than the first Fresnel zone [6] and varying slowly are correctly reconstructed. By ignoring refraction the algorithm is limited to low contrast flaws. Better algorithms that take into account refraction and diffraction exist [7] and will be studied in future work.

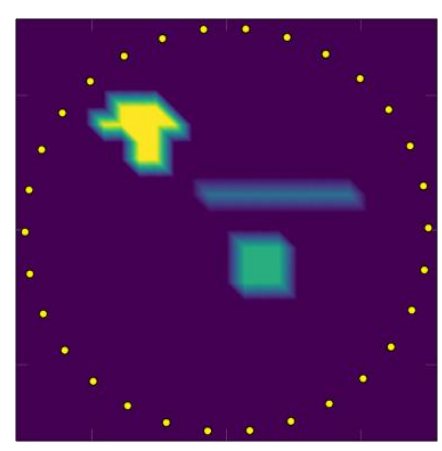

(a)

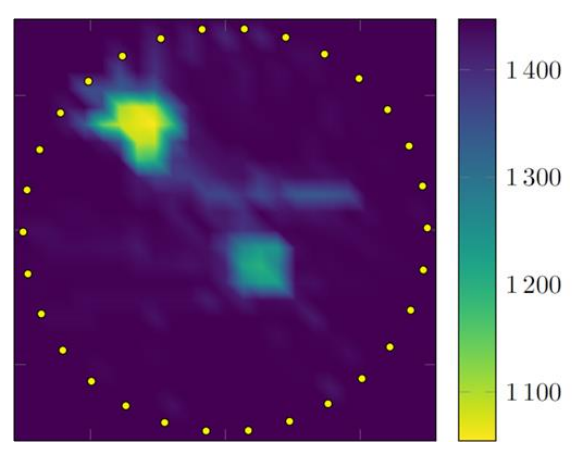

(b)

Figure 1: (a) Configuration; (b) Result after 1 iteration.

A simulation respecting these assumptions (i.e. no refraction and diffraction) was conducted (see Figure 1). The configuration on Figure 1a shows 30 sensors (which are depicted by yellow points) and three different defects. The image values matrix has a size of $23 \times 23$ pixels. Input data is a set of time-of-flight values deduced from dispersion curves of the $A_{0}$ mode at $30 \mathrm{kHz}$ propagating in a $2 \mathrm{~mm}$ thick plate. The absolute group velocity is $1447 \mathrm{~m} / \mathrm{s}$ when waves propagate in the healthy part of the plate and $1300 \mathrm{~m} / \mathrm{s}, 1200 \mathrm{~m} / \mathrm{s}$ and $1056 \mathrm{~m} / \mathrm{s}$ when they propagate in defect zones (i.e. zones of reduced thickness which can illustrate corrosion phenomenon). In practice, image smoothing is interesting since corrosion and pixels will unlikely be superimposed on each other. That is why Figure 1 and the experimental image on Figure $3 \mathrm{~b}$ are smoothed by performing interpolation.

\section{Experimental Setup}

The time-of-flight algorithm described in the previous section helped, among others things, to design an experimental setup (see Figure 2). The setup is composed of a computer running LabView which controls an oscilloscope and a multiplexer; a generator which is synchronized with the oscilloscope by a trigger; an amplifier; a filter and finally the piezoelectric transducers attached to the $2 \mathrm{~mm}$ thick aluminum plate. Phenyl salicylate (SALOL) is used to bond localized thin calibrated aluminum layers on the plate. For experimental purposes, these layers will serve as an easily removable defect with a somewhat similar effect than corrosion on wave velocity due to thickness change. 


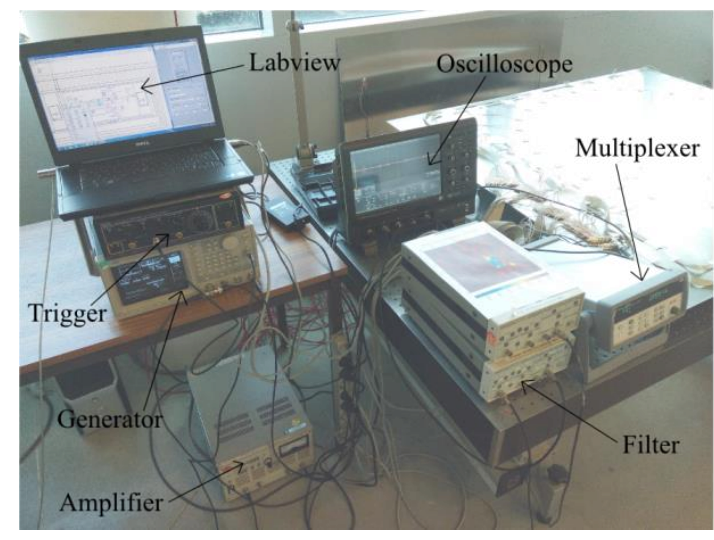

(a)

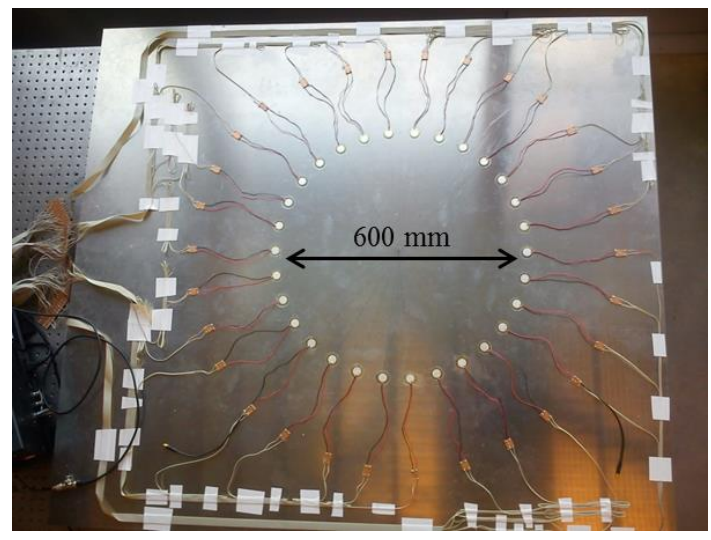

(b)

Figure 2: (a) Experimental setup; (b) 30 piezoelectric transducers ( $\varnothing 18 \mathrm{~mm}$ ) stuck on the $2 \mathrm{~mm}$ thick aluminum plate.

\section{Active experimental image}

Figure 3 shows an experimental active absolute time-of-flight tomography image (Figure 3b) which results from the algorithm described in Section 2. In practice, reversible flaws (Figure 3a) were used by adding a thin aluminum plate glued (with SALOL) on the plate to be inspected. The term 'Absolute tomography' means that data only comes from current signals. This way, the method should be quite robust as baseline signals are never used. Input data was obtained by emitting a 1.5 cycle tone-burst at $177.5 \mathrm{kHz}$ to the piezoelectric transducers. Time-of-flights from first $\mathrm{S}_{0}$ wave packet were identified by the algorithm for each pair of sensors. The two flaws of Figure $3 a$ are easily distinguishable on Figure 3b. Experimentally, there were local adhesive disbonds between aluminum plates, zones with poor SALOL adhesion and fluctuations in the thickness of the SALOL. All of those reasons partly explain why the reconstructed defects do not fit exactly the real lines of rectangular and circular aluminum plates.

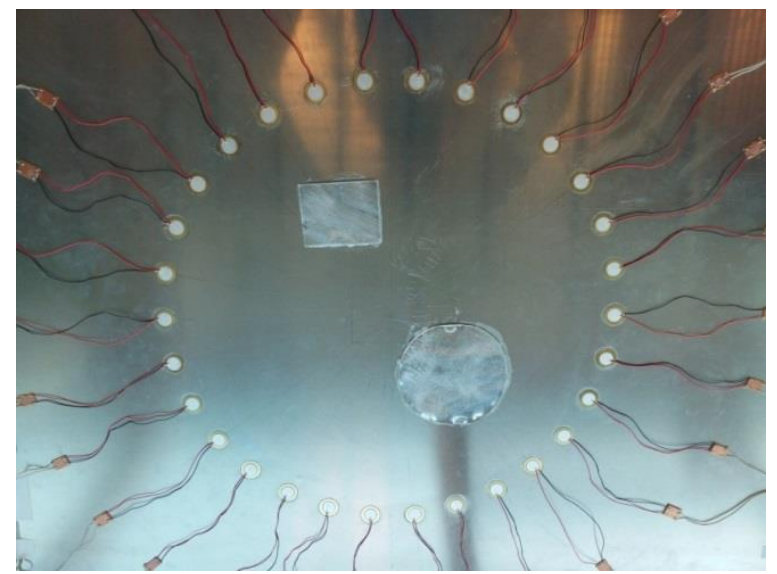

(a)

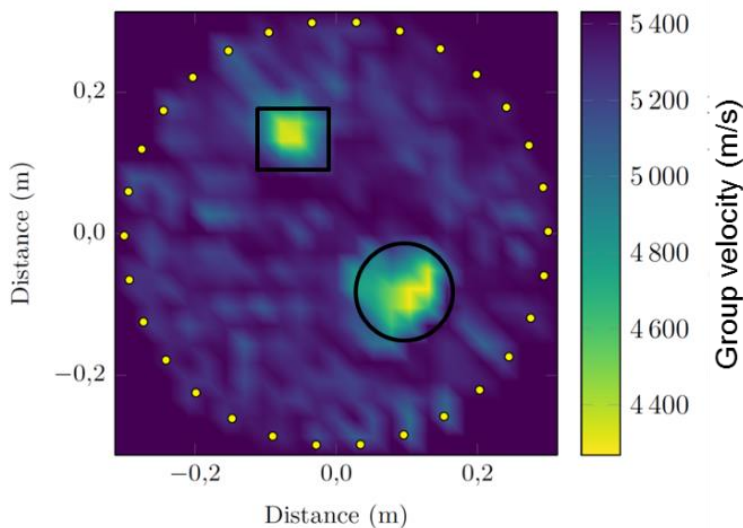

(b)

Figure 3: (a) Experimental configuration; (b) Absolute time-of-flight tomography (smoothed velocity map).

\section{Passive experimental results}

The input data necessary to obtain the tomography images is a set of time-of-flight values, as presented in previous sections. This Section aims at assessing the retrieval of this kind of data from passive 
signals. Equation 1 shows the cross-correlation formulation with $\vec{u}$ the displacement field at the point $\vec{x}$ and $\vec{v}$ the displacement field at the point $\overrightarrow{x^{\prime}}$. Every passive signal obtained in this Section results from cross-correlation of ambient noise recorded during the amount of time necessary (10 seconds in our experiments) for it to converge towards Green's functions.

$$
\underline{\underline{C}}_{\vec{u}, \vec{v}}\left(t, \vec{x}, \overrightarrow{x^{\prime}}\right)=\lim _{T \rightarrow \infty} \frac{1}{T} \int_{0}^{T} \vec{u}(\tau, \vec{x}) \vec{v}\left(t+\tau, \overrightarrow{x^{\prime}}\right)^{T} d \tau
$$

\subsection{Piezoelectric sensors (PZT)}

A comparison between active and passive signals, obtained thanks to cross-correlation of ambient elastic noise, is presented in Figure 4.

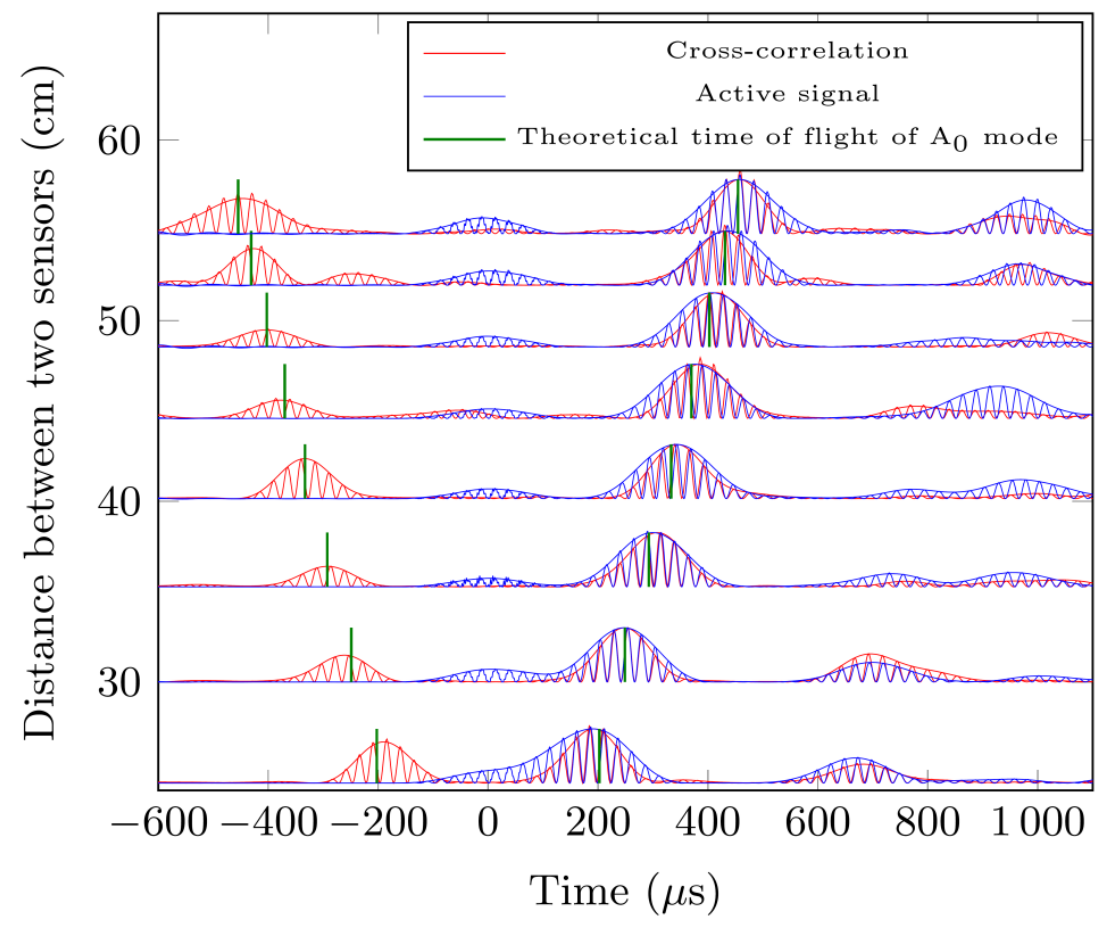

Figure 4: Comparison between active and cross-correlation signals.

Eight cycles tone-burst at $20 \mathrm{kHz}$ were generated in order to obtain the active signals shown in Figure 4. The elastic noise used for the passive signals was produced by spraying compressed air on the aluminum plate surface. The absolute values of those signals are plotted on Figure 4 for several distances between sensors. This way, the propagation of the first wave packet is shown for each active signal. Passive signals show a satisfactory reconstruction of Green's functions at least for the first wave packet. Indeed, the result is symmetrical (because of acoustic reciprocity between the two observation points) and first wave packets are adequately superimposed on passive and active signals. It is therefore possible to get time-of-flight from passive signals. This is a reason to think that passive tomography is promising. We can notice in the passive signals some parasite wavelets caused by the spatial distribution of the ambient noise [8,9]. Indeed, the more the ambient structural noise is spatially uniformly distributed the better the convergence towards Green's functions will be.

Figure 5 shows that it is possible to obtain time-of-flight on passive signals for higher frequencies, of at least $250 \mathrm{kHz}$ (for the ambient noise created by spraying the air on the aluminum plate). Here, the $S_{0}$ as well as $A_{0}$ modes can be detected in Figure 5 but it is not possible to correctly identify the time-of-flight for $A_{0}$. Indeed, $S_{0}$ reflections arrive at the same time as the first $A_{0}$ wave packet. 
[Type text]

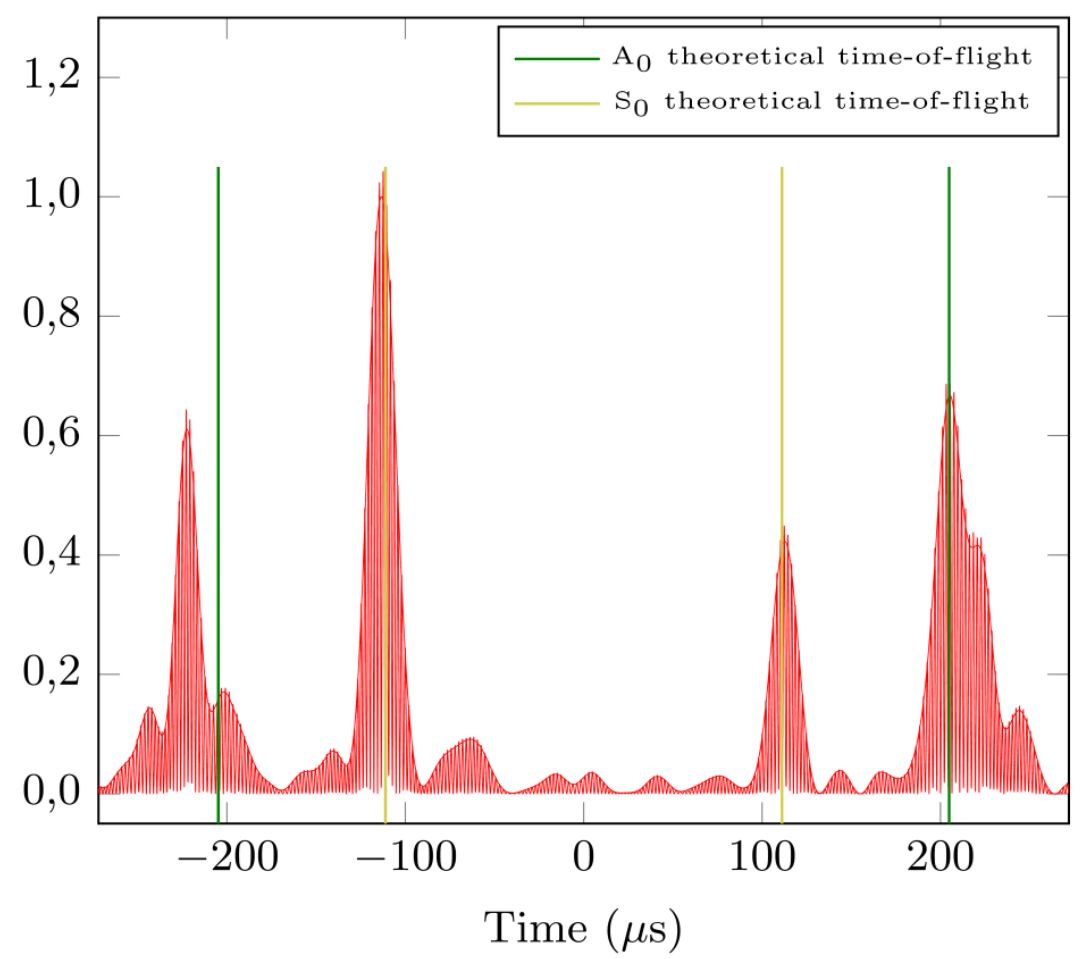

Figure 5; Reconstruction of passive signals for high frequencies - theoretical time-of-flights for S0 and $\mathrm{AO}$ at $250 \mathrm{kHz}$.

\subsection{Fiber Bragg Gratings (FBG)}

It has already been demonstrated that FBGs are able to detect guided waves emitted by piezoelectric transducers [10,11]. Here we perform passive experimental measurements using only fiber Bragg gratings sensors. With such passive measurements, PZT transducers for elastic wave emission would no longer be necessary, reducing the intrusiveness of the sensors in the structure. 
[Type text]

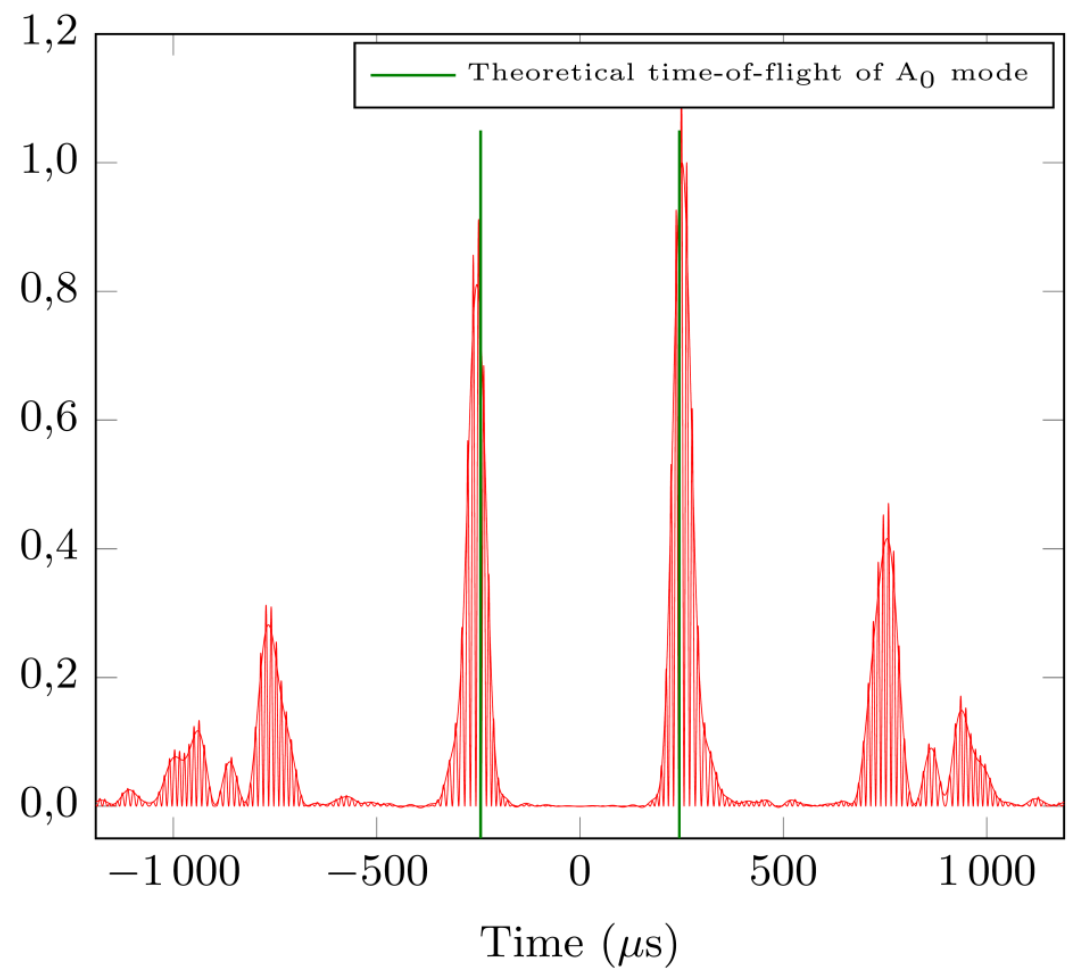

Figure 6: Reconstruction of passive signals with 2 fiber Bragg Gratings bonded on the surface of a $2 \mathrm{~mm}$ thick aluminum plate.

Elastic noise is generated in the structure using the same setup as in previous sections: by spraying compressed air on the aluminum plate surface. Figure 6 shows the passive reconstruction of the $A_{0}$ mode at $40 \mathrm{kHz}$ between FBGs bonded on the surface of a $2 \mathrm{~mm}$ thick aluminum plate and spaced $400 \mathrm{~mm}$ apart from each other.

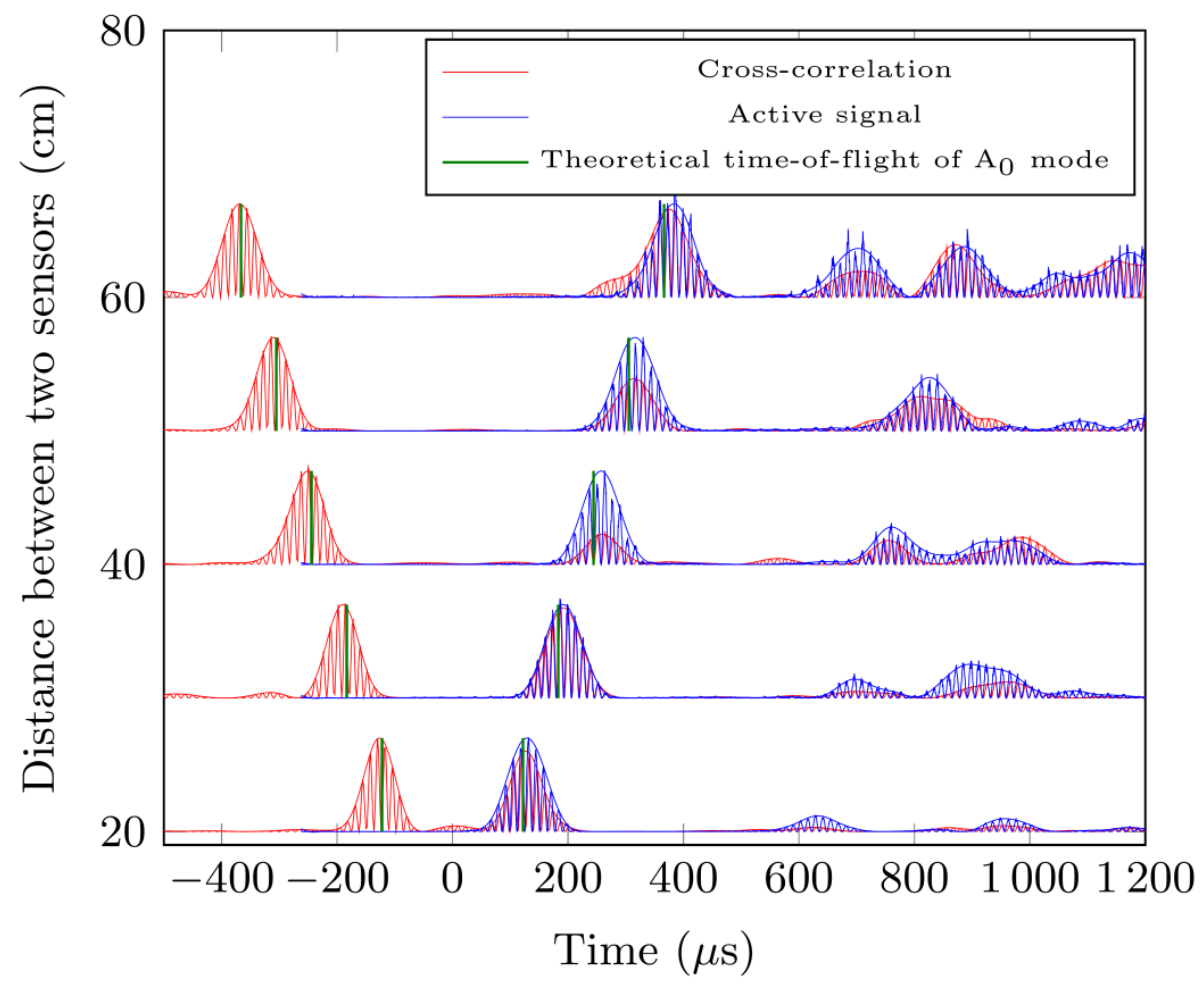

Figure 7: Reconstruction of passive signals between a piezoelectric transducer and a fiber Bragg grating. Comparison between active and cross-correlation signals. 
This demonstrates the feasibility of passive reconstruction using FBGs only. Indeed, the theoretical timeof-flight values perfectly match the maximum of the first wave packet ( $A_{0}$ mode). Figure 7 shows the passive reconstruction (in red) between a FBG and a PZT for several distances between both sensors. The PZT is used here only as sensor of the elastic noise, not to emit the guided waves. It has been chosen for convenience in this experiment because it is easier to move than the FBG. It confirms that the first wave packet is well reconstructed and that it is possible to correctly identify its time-of-flight with passive measurements using only FBG. In Figure 7, in the same way as in Figure 4, we can see that the first wave packets are adequately superimposed on passive and active signals.

\section{Conclusion}

This paper has shown that it is possible to image defects experimentally by using piezoelectric transducers and a time-of-flight tomography algorithm without the need of baseline signals. Active experimental tomography using guided wave data was performed in this paper. It was also shown that it is possible to detect time-of-flight by using cross-correlation of ambient noise with piezoelectric transducers as well as with fiber Bragg gratings. Passive experimental tomography is the next step of our study by merging the results obtained in these two domains.

\section{References}

[1] Croxford, A., P. Wilcox, B. Drinkwater and G. Konstantinidis. 2007. "Strategies for guided-wave structural health monitoring," Proc. R. Soc. A, 463: 2961-2981.

[2] Weaver, R. L. and O. I. Lobkis. 2001. "Ultrasonics without a Source: Thermal Fluctuation Correlations at MHz Frequencies," Phys. Rev. Lett., 87(13): 134301.

[3] Sabra, K. G., E. S. Winkel, D. A. Bourgoyne, B. R. Elbing, S. L. Ceccio, M. Perlin and D. R. Dowling. 2007. "Using cross correlations of turbulent flow-induced ambient vibrations to estimate the structural impulse response. Application to structural health monitoring," J. Acoust. Soc. Am., 121(4): 1987-1995.

[4] Moulin, E., N. Abou Leyla, J. Assaad and S. Grondel. 2009. "Applicability of acoustic noise correlation to Structural Health Monitoring in non-diffuse field conditions," Appl. Phys. Lett., 95: 094104.

[5] Kak, A. and M. Slaney. 1988. Principles of Computerized Tomographic Imaging. IEEE Press, New York, pp. 275.

[6] Williamson, P. R. 1991. "A guide to the limits of resolution imposed by scattering in ray tomography," Geophysics, 56(2): 202-207.

[7] Huthwaite, P. and F. Simonetti. 2013. "High-resolution guided wave tomography," Wave Motion, 50: 979-993.

[8] Chehami, L., E. Moulin, J. De Rosny, C. Prada, J. Assaad and F. Benmeddour. 2015. "Experimental study of passive defect detection and localization in thin plates from noise correlation", Proc. of ICU 2015, Metz (France).

[9] Colombi, A., L. Boschi, P. Roux and M. Campillo. 2014. "Green's function retrieval through crosscorrelations in a two-dimensional complex reverberating medium," Journal of the Acoustical Society of America, 135: 1034-1043.

[10] Betz, D., G. Thursby, B. Culshaw and W. Staszewski. 2003. "Acousto-ultrasonic sensing using fiber Bragg gratings", Smart Materials and Structures, 12: 122-128.

[11] Botsev, Y., E. Arad, M. Tur, I. Kressel, U. Ben-Simon, S. Gail and D. Osmont. 2008. "Structural Health Monitoring using an embedded PZT-FBG ultrasonic sensor array", Proceedings of the Fourth European Workshop on Structural Health Monitoring, Cracow (Poland). 